\title{
Clinical and histological evaluation of large macular hole surgery using the inverted internal limiting membrane flap technique
}

This article was published in the following Dove Press journal:

Clinical Ophthalmology

16 December 2016

Number of times this article has been viewed

\section{Satoru Kase \\ Wataru Saito \\ Shohei Mori \\ Michiyuki Saito \\ Ryo Ando \\ Zhenyu Dong \\ Tomohiro Suzuki \\ Kousuke Noda \\ Susumu Ishida}

Department of Ophthalmology, Hokkaido University Graduate School of Medicine, Sapporo, Japan
Correspondence: Satoru Kase

Department of Ophthalmology, Hokkaido University Graduate School of Medicine,

N-I5, W-7, Kita-ku, Sapporo

060-8638, Japan

$\mathrm{Tel}+8 \mathrm{I}$ II 7065944

Fax +8I II 7065948

Email kaseron@med.hokudai.ac.jp
Purpose: The aims of this study were to analyze optical coherence tomography (OCT) imaging of large macular holes (MHs) treated with inverted internal limiting membrane (ILM) flap technique and to perform a histological examination of an ILM-like membrane tissue obtained during vitrectomy.

Patients and methods: This is a retrospective observational case study. Nine patients, comprising of five males and four females, showing large and myopic MHs, underwent pars plana vitrectomy (PPV) with inverted ILM flap technique assisted by brilliant blue G (BBG) staining. Ophthalmological findings including visual acuity and OCT were investigated based on medical records. Formalin-fixed paraffin-embedded tissue section of an ILM-like membrane was submitted for immunohistochemistry with glial fibrillary acidic protein (GFAP).

Results: ILM was clearly stained with BBG in eight patients, whereas the ILM in one case revealed no staining with BBG during PPV. Visual acuities improved to $>0.2$ LogMAR in six patients. The complete closure of MH following PPV with inverted ILM technique was eventually achieved in all patients determined by OCT imaging (100\%). Only one patient showed recovery of ellipsoid zone and interdigitation zone following the surgery. Elongation of outer nuclear layer was noted in three eyes. The ILM-like membrane not stained with BBG histologically revealed an amorphous structure admixed with GFAP-positive mononuclear cell infiltration.

Conclusion: PPV with inverted ILM flap technique achieved $100 \%$ closure rates with favorable configuration at an initial surgery in large MHs. Our histopathological data also suggest that even BBG staining-negative membrane may be a useful material for autologous transplantation to the hole.

Keywords: inverted ILM flap, glial cells, OCT, macular hole, histopathology

\section{Introduction}

Internal limiting membrane (ILM), the basement membrane of retinal Müller cells, is the structural interface between the vitreous and retina. Histologically, ILM tissues isolated from patients with macular holes (MHs) revealed amorphous linear structure in which glial cells, hyalocytes, and myofibroblast-like cells could be intermingled., ${ }^{1,2}$ Today, brilliant blue G (BBG) is well known to be a useful agent to stain ILM with blue coloration and to facilitate ILM peeling. ${ }^{3}$ ILM removal in MH surgeries relieves tangential traction around the holes, increases $\mathrm{MH}$ closure rates, and contributes to reduced frequency of epiretinal membrane formation. ${ }^{4}$ Thus, ILM peeling during vitrectomy has become one of the pivotal procedures in surgical intervention for MHs.

However, vitreoretinal surgeons cannot always close every $\mathrm{MH}$ using vitrectomy with conventional ILM peeling. Especially, surgical interventions for large MHs, 
measuring $>400 \mu \mathrm{m}$ in the base diameter, and for highmyopic MHs are challenging because of their low closure rates. ${ }^{5}$ Sometimes, such holes could be closed with exposure of the retinal pigment epithelium to the vitreous space, resulting in postoperative poor visual acuity. ${ }^{6}$ In order to improve the hole closure rate, inverted ILM flap technique has recently been applied to patients with refractory MHs. Indeed, this technique includes isolation of ILM tissues, which are not completely peeled off but placed on the hole. This technique is also useful for closure of a large $\mathrm{MH}$ secondary to uveitis. ${ }^{7}$ However, it is largely unknown whether it is possible to use BBG-negative membranes as materials for autologous transplantation in order to facilitate the hole closure. In addition, it has not been clarified how the inverted ILM flap technique leads to morphological changes following the MH closure and to postoperative visual outcomes.

The aim of this study is to analyze optical coherence tomography (OCT) imaging of large MHs treated with inverted ILM flap technique. This study further performed a histological examination of an ILM-like membrane tissue obtained during vitrectomy.

\section{Patients and methods}

\section{Patients}

This retrospective observational study enrolled nine patients with full-thickness large MHs. The diagnostic criterion for large $\mathrm{MH}$ includes the minimum diameter of the holes $>400 \mu \mathrm{m}$ determined by OCT imaging at an initial presentation. High-myopic $\mathrm{MH}$ is identified as follows: spherical equivalent $>-7.0 \mathrm{D}$ and the ocular axis $>26 \mathrm{~mm}$ determined by A-scan ultrasonography and/or intraocular lens (IOL) master. Patients aged $<20$ years were excluded. This study also excluded cases showing a minimum $\mathrm{MH}$ diameter of $<400 \mu \mathrm{m}$, the existence of proliferative vitreoretinopathy or other retinal breaks, $\mathrm{MH}$ retinal detachment, and medical history of having ocular surgeries. All patients underwent $23 \mathrm{G}$ or $25 \mathrm{G}$ pars plana vitrectomy (PPV) with/ without phacoemulsification and IOL implantation, conducted by SK and WS in Hokkaido University Hospital or Teine Keijinkai Hospital between June 2013 and March 2015. If the posterior vitreous detachment (PVD) was not present, the surgeon manually conducted artificial PVD formation. BBG was injected to visualize ILM in all patients examined. After visualization of ILM by BBG staining, ILM was peeled but the ILM around MH was not completely removed. The ILM tissues were left as much as possible, which were placed within the hole based on the previous reports. ${ }^{8,9}$ The ILM tissues beyond the hole within the arcade were then peeled off. SF6 gas, with the concentration of $16 \%-20 \%$, was injected following fluid/air exchange. The patients were kept in prone position for a few days after the surgeries. Postoperative ophthalmological findings, including best-corrected visual acuities, dilated fundus examination, and OCT imaging (CIRRUS HD-OCT model 4000 [Carl Zeiss Meditec, Osaka, Japan]), were recorded before and 1,3 , and 6 months after surgery. This study was approved by the institutional review board (IRB) of Hokkaido University Graduate School of Medicine (IRB number: 15-0068), and the study adhered to tenets set forth in the Declaration of Helsinki. Informed consent was obtained from patients.

\section{Histology and immunohistochemistry}

The ILM tissue was excised during vitrectomy, which was immediately fixed with $4 \%$ paraformaldehyde in an operating room. Formalin-fixed, paraffin-embedded tissue sections of $5 \mu \mathrm{m}$ thickness were made. The slides were dewaxed, rehydrated, and rinsed in phosphate-buffered saline (PBS) twice for 10 minutes and were submitted for hematoxylin and eosin staining and periodic acid Schiff (PAS) staining to confirm the presence of ILM. The slides were further submitted for immunohistochemistry. As a pretreatment, microwave-based antigen retrieval was performed in $10 \mathrm{mM}$ citrate buffer ( $\mathrm{pH} 6.0$ ). These slides were incubated with $3 \%$ hydrogen peroxide for 10 minutes and then with normal goat serum for 30 minutes. Sections were incubated with anti-glial fibrillary acidic protein (GFAP; dilution 1:50; Dako, Tokyo, Japan) antibody at $4^{\circ}$ overnight. Positive signals were visualized using diaminobenzidine as a substrate. Slides were examined using a Keyence BZ-9000 (Keyence, Osaka, Japan) microscope.

\section{Statistical analysis}

Wilcoxon-signed rank test was applied for the evaluation of differences in visual acuities before and after the surgeries at the final presentation. The level of significance for the test was $P<0.05$.

\section{Results}

Nine patients comprising five males and four females were examined in this study. The patients' age ranged from 51 to 71 years (mean: 64.8 years). Four right eyes and five left eyes were involved. Table 1 summarizes the clinical profiles of patients with $\mathrm{MH}$ examined in this study. All the nine patients revealed large $\mathrm{MHs}$, consisting of five idiopathic $\mathrm{MHs}$, one large $\mathrm{MH}$ secondary to branch retinal vein occlusion, and three high-myopic MHs. Eight patients showed phakic eyes having incipient or mild cataract. Eight patients underwent 


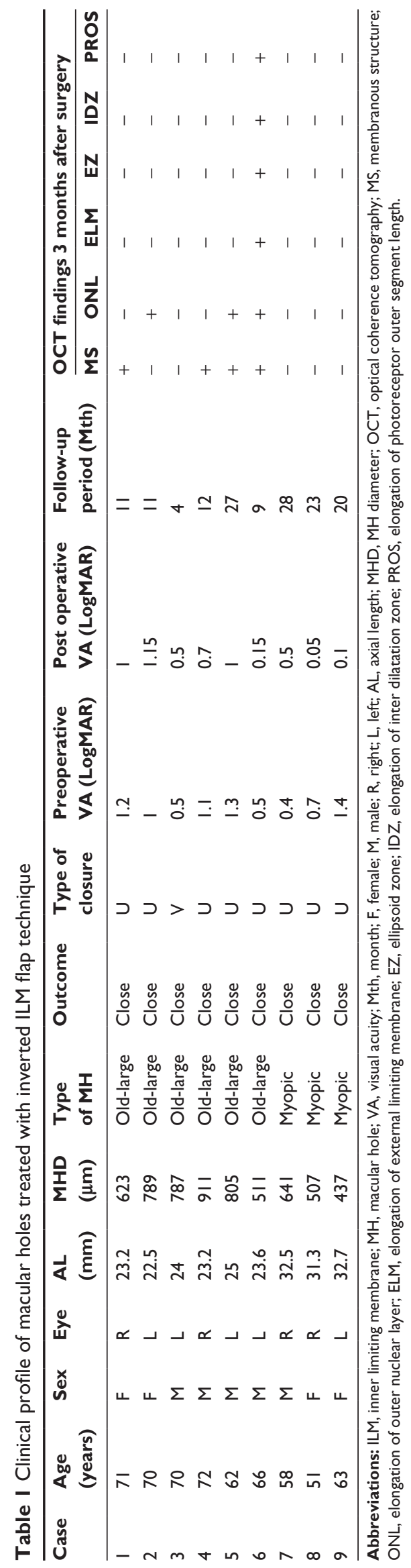

PPV together with phacoemulsification and IOL implantation, while one patient already had a pseudophakic eye before surgery. ILM was clearly stained with BBG in eight patients (Figure 1A-D), whereas the ILM in Case 2 (Table 1) revealed no staining with BBG even after completion of artificial PVD formation (Figure 1E-G). No surgery-related complications were noted in all the patients examined. The follow-up periods ranged from 4 to 28 months (mean: 16.1 months). Preoperative and postoperative best-corrected visual acuities at the final visit (LogMAR) were $0.90 \pm 0.38$ and $0.57 \pm 0.41$, respectively. There was no significant improvement in postoperative visual acuity $(P=0.10)$, although visual acuities improved to $>0.2 \operatorname{LogMAR}$ in six patients.

\section{Changes in OCT findings before and after surgery}

OCT findings were available 1-3 days after the surgery under gas tamponade in three eyes, in which all the patients examined showed findings suggestive of favorable closure. The complete closure of MH following PPV with inverted ILM technique was eventually achieved in all patients determined by OCT imaging (100\%) by 1 month after surgery. In this study, after PPV with inverted ILM flap technique, the closure type was U-type (Figures 1D, H and 2A, B) and V-type (Figure 2C and D) in eight eyes and one eye, respectively. OCT detected linear configuration, consistent with transplanted ILM tissues, in four eyes 1 month after the surgery. Three out of nine eyes showed elongation of outer nuclear layer 3 months after the surgery. Only one patient (Case 6 in Table 1) showed recovery of ellipsoid zone and interdigitation zone, and elongation of photoreceptor outer segment length following the surgery. There are no significant changes in OCT finding between 3 and 6 months after the surgery.

\section{Histological findings}

The tissue of an ILM-like membrane was isolated during PPV in one patient with stage III MH (Case 2 in Table 1). The ILM-like membrane not stained with BBG revealed an eosinophilic amorphous structure in conjunction with mononuclear cell infiltration (Figure 3A). PAS staining showed negative for cell infiltration but positive for the amorphous structure, which is consistent with ILM (Figure 3B). The mononuclear cells revealed immunoreactivity for GFAP (Figure 3C).

\section{Discussion}

In this study, PPV with inverted ILM flap technique achieved $100 \%$ closure rates at the initial surgery in nine patients with 

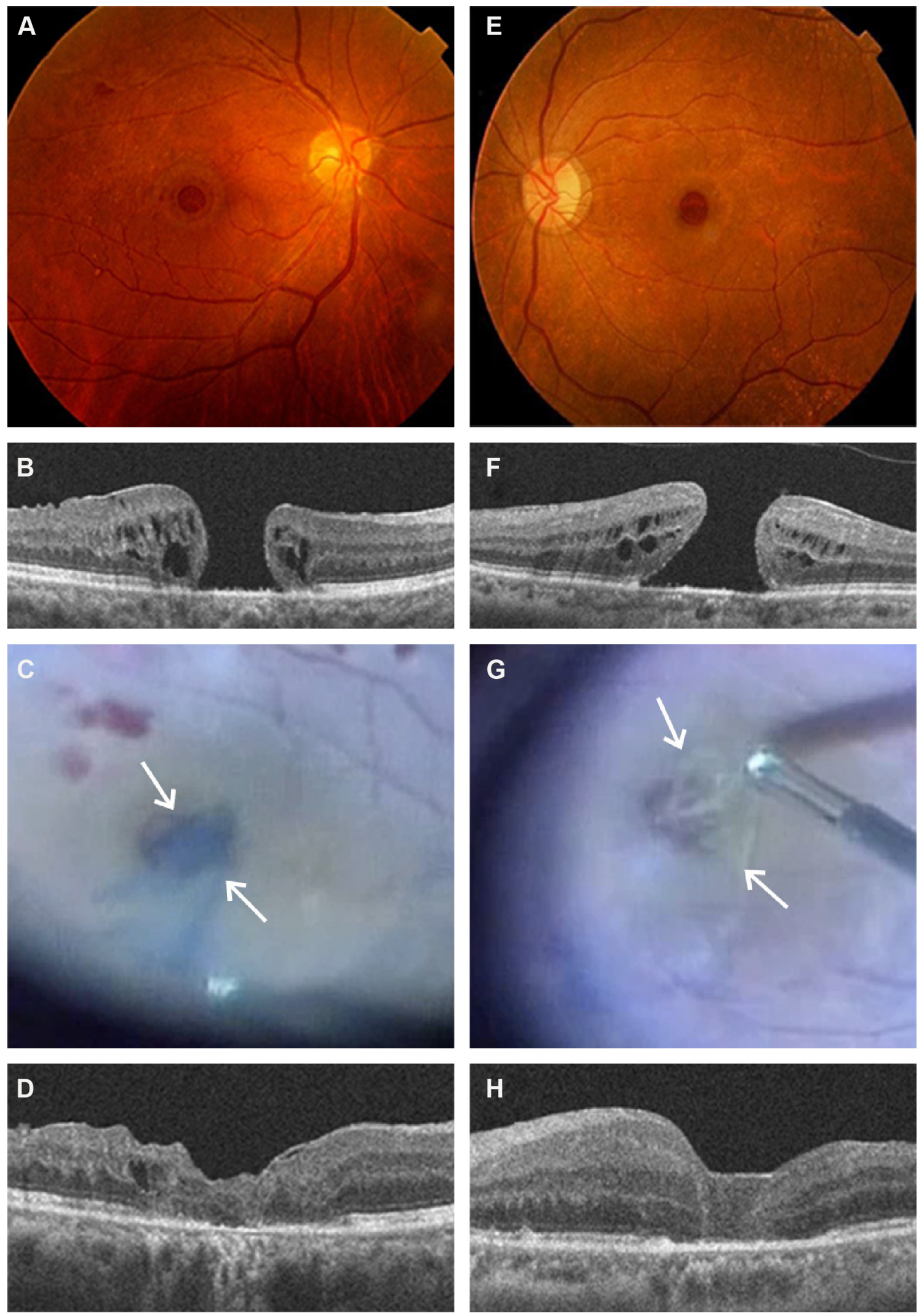

Figure I Representative two cases (Case I, A-D; Case 2, E-H) with old-large macular hole treated with pars plana vitrectomy and inverted internal limiting membrane (ILM) flap technique.

Notes: In Case I, a 7I-year-old female presents with Stage IV large macular hole (A), measuring a minimum diameter of $623 \mu \mathrm{m}$ (B). During vitrectomy, the ILM is stained with brilliant blue G (BBG), which was placed within the hole (C, arrows). Macular hole is successfully closed 3 months after the surgery (D). In Case 2, a 70 -year-old female presents with Stage III large macular hole (E), measuring a minimum diameter of $789 \mu \mathrm{m}(\mathbf{F})$. In intraoperative findings, ILM-like membrane without staining with BBG was placed within the hole in the vitrectomy (G, arrows). The macular hole is successfully closed 3 months after the surgery $(\mathbf{H})$ as shown in Case $\mathbf{I}$. 

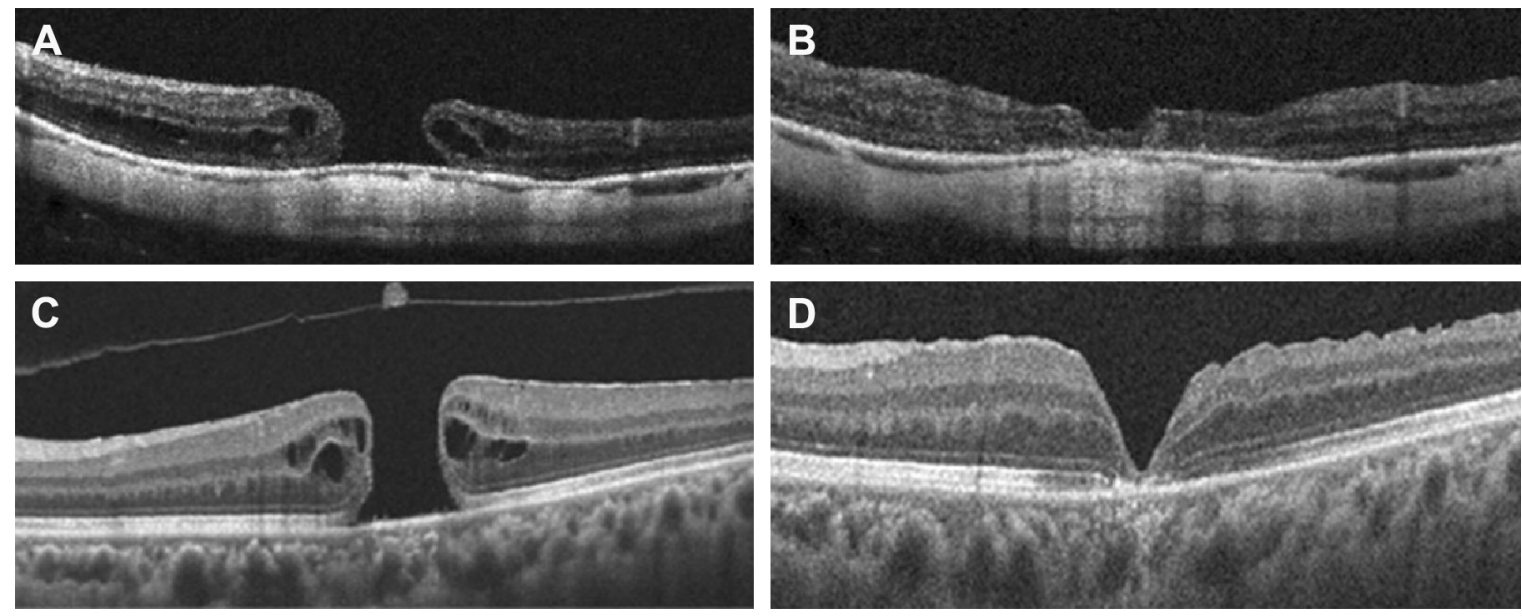

Figure 2 Other OCT findings before and after vitrectomy with inverted ILM flap technique.

Notes: OCT detects large macular hole in a patient with high myopia (A). Three months after the surgery, macular hole is successfully closed with a favorable configuration (B). The other patient reveals a large macular hole without high myopia (C). However, macular hole is closed with thin retinal tissues covering after the surgery (D). Abbreviations: OCT, optical coherence tomography; ILM, internal limiting membrane.

large MHs. Michalewska et $\mathrm{al}^{8}$ first described the initial closure rate of $98 \%$ by inverted ILM flap technique in the patients with large $\mathrm{MH}$. Kuriyama et $\mathrm{al}^{5}$ reported primary closure following the surgery in five eyes out of six eyes with highly myopic MH. Imai et $\mathrm{l}^{6}$ proposed OCT-based evaluation of $\mathrm{MH}$ closure following vitrectomy. Briefly, U-type closure is the most favorable closure type possibly leading to good postoperative visual acuity. Next, V-type shows notch configuration covered with thin retinal tissue. Finally, W-type is not favorable closure, so-called open-closed $\mathrm{MH}$, exposing retinal pigment epithelium to the vitreous space. This case series also proved ideal U-type closure, a successful closure configuration, in most eyes using the inverted ILM flap technique.

In this study, the ILM-like membrane was partially removed in one eye showing no staining with $\mathrm{BBG}$, which was submitted for pathological analyses. Histologically, the ILM-like membrane contained an amorphous linear structure that is PAS positive, which is typically observed in the ILM tissue. Moreover, a variety of mononuclear cells infiltrated the tissue. These cells were positive for GFAP, suggesting glial cell infiltration. Uemoto et $\mathrm{al}^{10}$ reported that BBG-positive ILM isolated from $\mathrm{MH}$ and epiretinal membrane revealed a membrane with few cell infiltration based on pathological analysis. Therefore, the BBG-negative case shown in this report may represent more glial cell infiltration within the ILM tissue than ILM stained with BBG. As shown in Figure 1, the postoperative OCT findings were similar to those transplanted by common BBG-positive ILM tissues. Kuriyama et $\mathrm{al}^{5}$ reported that epiretinal membranes/ILM tissues were transplanted in the hole during vitrectomy in three eyes among six high myopic eyes. Taken together, our data also suggest that even BBG staining-negative ILM-like membrane can be safely used as a material for autologous transplantation to the hole.

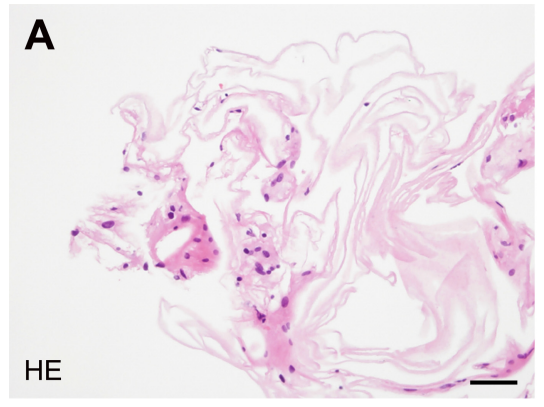

B

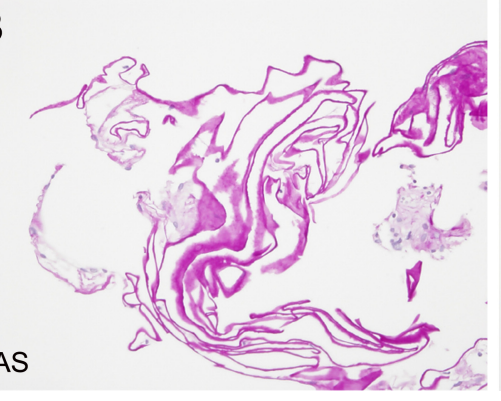

C

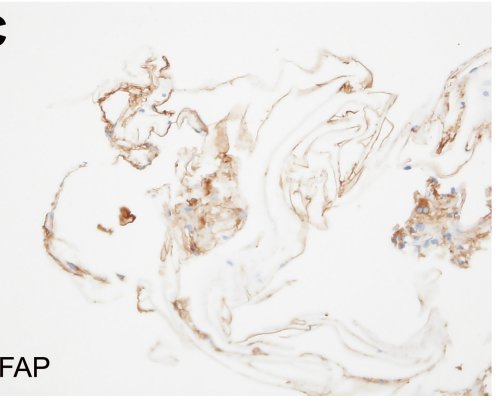

Figure 3 Hematoxylin and eosin (HE) staining (A), periodic acid Schiff (PAS) staining (B), and immunohistochemistry for glial fibrillary acidic protein (GFAP) (C) in the internal limiting membrane (ILM)-like membrane in a patient with large macular hole (Case 2).

Notes: The ILM not stained with brilliant blue G reveals eosinophilic amorphous structures admixed with mononuclear cell infiltration (A). PAS staining shows negative for cell infiltration but positive for the amorphous structure in consistence with ILM (B). The mononuclear cells are GFAP-positive (C). (A) Bar equals $50 \mu \mathrm{m}$. 
The mechanisms underlying the hole closure using inverted ILM flap technique remain largely unknown. It has been speculated that ILM peeling may induce retinal gliosis, leading to the formation of a bridge between the deficit of retinal tissues in the hole. ${ }^{8}$ This study indicated that the ILMlike membrane including GFAP-positive retinal glial cells contributed to the hole closure with a favorable configuration determined by OCT. These results suggest that glial cells placed on the hole may produce intermediate filaments and provoke tissue remodeling within the $\mathrm{MH}$.

The OCT demonstrated no recovery of deficit of ellipsoid zone and no elongation of photoreceptor outer segment length in eight out of nine patients examined, although six patients showed improvement of $>0.2$ LogMAR visual acuities. In contrast, no patients demonstrated deterioration of $>0.2$ LogMAR visual acuities. de Sisternes et al ${ }^{11}$ addressed that recovery of visual acuity was correlated with preoperative degree of the ellipsoid zone deficit in patients with $\mathrm{MH}$. We recently showed that elongation of photoreceptor outer segment length following MH surgery correlated with postoperative visual acuities. ${ }^{12}$ However, these results are not consistent with the present study, indicating that the recovery of visual acuity did not depend on preoperative severity of ellipsoid zone and photoreceptor damage in patients with large MH treated with inverted ILM flap technique. In this study, elongation of outer nuclear layer and external limiting membrane was noted in a few patients, indicating that alteration of visual fixation may correlate with the recovery of visual acuity following the surgery in selected cases. Further studies are needed to analyze the mechanisms underlying postoperative recovery of visual acuity in inverted ILM flap technique.

\section{Conclusion}

PPV with inverted ILM flap technique achieved $100 \%$ closure rates with favorable configuration at an initial surgery in large MHs. Our data also suggest that even BBG staining-negative membrane may be one of useful materials for autologous transplantation to the hole.

\section{Acknowledgment}

The authors thank Yuki Hashimoto for his technical assistance in measuring macular hole diameters. No financial support was received for this submission.

\section{Disclosure}

The authors report no conflicts of interest in this work.

\section{References}

1. Kase S, Saito W, Ohno S, Ishida S. Cyclo-oxygenase-2 expression in human idiopathic epiretinal membrane. Retina. 2010;30(5):719-723.

2. Schumann RG, Eibl KH, Zhao F, et al. Immunocytochemical and ultrastructural evidence of glial cells and hyalocytes in internal limiting membrane specimens of idiopathic macular holes. Invest Ophthalmol Vis Sci. 2011;52(11):7822-7834.

3. Steel DH, Dinah C, Madi HA, White K, Rees J. The staining pattern of brilliant blue $\mathrm{G}$ during macular hole surgery: a clinicopathologic study. Invest Ophthalmol Vis Sci. 2014;55(9):5924-5931.

4. Vaziri K, Schwartz SG, Kishor KS, et al. Rates of reoperation and retinal detachment after macular hole surgery. Ophthalmology. 2016; 123(1):26-31.

5. Kuriyama S, Hayashi H, Jingami Y, Kuramoto N, Akita J, Matsumoto M. Efficacy of inverted internal limiting membrane flap technique for the treatment of macular hole in high myopia. Am J Ophthalmol. 2013;156:125-131.e1.

6. Imai M, Iijima H, Gotoh T, Tsukahara S. Optical coherence tomography of successfully repaired idiopathic macular holes. Am J Ophthalmol. 1999;128(5):621-627.

7. Hirano M, Morizane Y, Kawata T, et al. Case report: successful closure of a large macular hole secondary to uveitis using the inverted internal limiting membrane flap technique. BMC Ophthalmol. 2015;15:83.

8. Michalewska Z, Michalewski J, Adelman RA, Nawrocki J. Inverted internal limiting membrane flap technique for large macular holes. Ophthalmology. 2010;117(10):2018-2025.

9. Michalewska Z, Michalewski J, Dulczewska-Cichecka K, Adelman RA, Nawrocki J. Temporal inverted internal limiting membrane flap technique versus classic inverted internal limiting membrane flap technique: a comparative study. Retina. 2015;35(9):1844-1850.

10. Uemoto R, Nakasato-Sonn H, Meguro A, Ito N, Yazama F, Mizuki N. Staining internal limiting membrane with a mixture of BBG and sodium hyaluronate. Br J Ophthalmol. 2013;97(6):690-693.

11. de Sisternes L, Hu J, Rubin DL, Leng T. Visual prognosis of eyes recovering from macular hole surgery through automated quantitative analysis of spectral-domain optical coherence tomography (SD-OCT) scans. Invest Ophthalmol Vis Sci. 2015;56(8):4631-4643.

12. Hashimoto Y, Saito W, Fujiya A, et al. Changes in inner and outer retinal layer thicknesses after vitrectomy for idiopathic macular hole: implications for visual prognosis. PLoS One. 2015;10(8):e0135925.
Clinical Ophthalmology

\section{Publish your work in this journal}

Clinical Ophthalmology is an international, peer-reviewed journal covering all subspecialties within ophthalmology. Key topics include: Optometry; Visual science; Pharmacology and drug therapy in eye diseases; Basic Sciences; Primary and Secondary eye care; Patient Safety and Quality of Care Improvements. This journal is indexed on Submit your manuscript here: http://www.dovepress.com/clinical-ophthalmology-journal

\section{Dovepress}

PubMed Central and CAS, and is the official journal of The Society of Clinical Ophthalmology (SCO). The manuscript management system is completely online and includes a very quick and fair peer-review system, which is all easy to use. Visit http://www.dovepress.com/ testimonials.php to read real quotes from published authors. 